Elena lakovou

\title{
Tragödie im Roman: Euripides-Rezeption in Heliodors Aithiopika
}

\begin{abstract}
This article examines to what extent Heliodorus' novel Aethiopica makes use of Euripides' plays as a source of inspiration within the literary milieu of the Second Sophistic, where Euripidean influences can be found stretched across rich literary, visual and performative evidence, both in the private and public domain. The article shows that Heliodorus not only adapts structural elements of Euripidean tragedies, but also depicts various characters bearing clear tragic and Euripidean traits which he then subverts. Moreover, the analysis discusses for the first time the reception of Euripides' fragmentary tragedies in the Aethiopica.
\end{abstract}

\section{Einleitung}

Die Kaiserzeit zeigte allgemein ein reges Interesse an den attischen Dramen, insbesondere an denen des Euripides, sowohl im Schulunterricht und in rhetorischen Übungen (Progymnasmata) als auch in Theateraufführungen. ${ }^{1}$ Davon ist auch Heliodors $^{2}$ Roman Aithiopika nicht ausgenommen, der gemeinhin in das zweite Viertel des

\footnotetext{
1 Dazu Mastronarde 2017, 13-21 und oben der Beitrag von Martin Hose in diesem Band. Zur Texttradition der euripideischen Tragödien vom 4. Jh. v.Chr. bis zum 8 Jh. n. Chr. siehe ausführlich Carrara 2009. Allgemein zum Bildungswesen in der Antike siehe Marrou 1948. Zur griechischen Schulbildung in der hellenistischen und römischen Welt siehe Cribiore 2001a. Scourfield 2007, 5 weist darauf hin, dass zentralen literarischen Texten große Bedeutung beigemessen wurde, indem sie als Allgemeingut galten und in den Schulkanon aufgenommen wurden: „Under the Roman Empire, the governing classes maintained their identity partly through their participation in a highly conservative education system which stressed facility in language from grammar to sophisticated rhetorical composition, and in which the close study of specific literary texts was central.“ Zur Rezeption des Euripides (anhand der Tragödie Iphigenie in Aulis) in der Kaiserzeit siehe Hall 2013. Allgemein zum griechischen und römischen Theater siehe Harrison u. Liapis 2013. Zu den Theateraufführungen in der römischen Kaiserzeit Jones 1993, 39-52.

2 Anders als die Romane des Antonios Diogenes, Chariton, Achilleus Tatios und Longos, die zu Beginn Auskünfte über den Autor und den Inhalt der Liebesgeschichte enthalten, stellt Heliodor erst am Ende seines Romans biographische Informationen über sich selbst vor. Eine Parallele zur Heliodors Sphragis

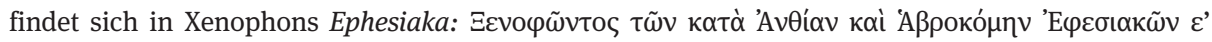

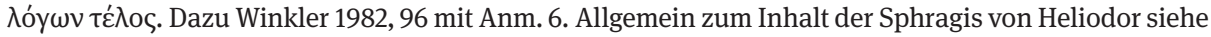
Hilton 2012, 195-219. Das historiographische Gewand des antiken Romans wird durch die Hinzufügung der Sphragis als literarisches Produkt hervorgehoben (vgl. Herodots programmatisches Proömium und Charitons Sphragis zu Beginn des Romans). Zur „historiographischen Pose“ Heliodors siehe Morgan 1982, 221- 265.
}

https://doi.org/10.1515/9783110677072-012 
4. n. Jh. datiert wird ${ }^{3}$ und zahlreiche Bezüge zu Euripides' Tragödien (in Form von Allusionen oder Abweichungen) aufweist.

Auch die anderen griechischen Romane enthalten intertextuelle Bezüge zu Euripides, ${ }^{4}$ aber nicht so zahlreich und durchdacht wie in Heliodors Aithiopika. Eine besondere Attraktivität dürften für Heliodor die ,gebrochenen' Charaktere des Euripides besessen haben, denen keine heroischen Züge wie bei Aischylos und Sophokles zugeschrieben werden. Sie sind Menschen, die ein alltägliches Leben führen und die sozialen und politischen Verhältnisse kritisch hinterfragen. ${ }^{5}$ Die Frauengestalten nehmen oftmals eine dynamische und aktive Haltung ein (z. B. Alkestis, Medea, Iokaste, Andromache, Makaria), während sich die männlichen Figuren auf marginale Rollen beschränken (z. B. Hippolytos, Iason, Orestes). ${ }^{6}$ Außerdem haben die Charaktere Schwächen und sind durch Affekt (pathos) geleitet, wie beispielsweise Herakles und Medea. ${ }^{7}$ Solche und andere entheroisierten Figuren setzt Heliodor in besondere Verbindung zu den Figuren seines Romans, sei es durch Allusionen, sei es durch Kontrastimitationen, wie das Folgende zeigen soll.

Nach einer kurzen Übersicht über die Romanhandlung soll der Fokus anhand ausgewählter Tragödien des Euripides auf die Aspekte gerichtet werden, welche den kreativen Umgang Heliodors mit Euripides aufzeigen. Dabei soll erörtert werden, inwiefern Heliodor nicht nur auf die tragischen Bearbeitungen verschiedener mythologischer Stoffe des Euripides rekurriert, sondern auch auf die uns nur fragmentarisch erhaltenen Tragödien Bezug nimmt. ${ }^{8}$

\section{Die Handlung der Aithiopika}

Im Mittelpunkt der insgesamt zehn Bücher umfassenden Aithiopika steht ein junges Paar namens Theagenes (aus Thessalien) und Charikleia (aus Ägypten). ${ }^{9}$ Ihre Lie-

3 So Mecella 2014, 638-646; vgl. auch Cerulli 2012, 15f.; Futre-Pinheiro 2014, 201-216; Ross 2014, 1-26; Hilton 2016, 25 -42. Bereits in den 90er Jahren wurden Heliodors Aithiopika ins 4. Jh. n. Chr. datiert: z.B. von Chuvin 1990, 321-325 und Morgan 1982, 221-265. Für eine frühere Datierung des Romans in die Mitte des 3. Jh. n. Chr. plädiert Bowie 2008, 32-35. Henrichs 2011, 312 merkt richtig an: „The case of Heliodoros reminds us that the papyri are not a panacea for all the problems besetting the chronology of the novel [...]. Only a new papyrus of Heliodoros from the third century or the first half of the fourth century could bring absolute certainty.“

4 Zur Gattung Roman siehe Holzberg 1996, 11-28 und zuletzt Whitmarsh 2018.

5 Vgl. Ar. Ra. 948-950, 956-967, 1051-1056, 1058-1062.

6 Hose 2008, 240.

7 Vgl. Ar. Ra. 1078-1088.

8 Zu den euripideischen Tragödien als Prätext für Heliodor siehe Billault 1998, 179-194; Paulsen 1992 sowie Lefteratou 2017. Zu den anderen Romanautoren siehe beispielsweise Hirschberger 2001, 157-186 sowie Scourfield 2010, 291-313 (Chariton); Mignonga 1997, 225-236 (Achilleus Tatios).

9 Die Namen der Protagonisten werden erst aus ihrem Gespräch bekannt (vgl. 1,8,3f.). Bis zu diesem Punkt der Erzählung werden die beiden Protagonisten als „Mädchen“ (кó

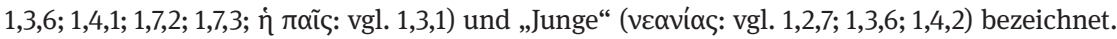


besgeschichte wird teils von den Romanfiguren selbst, teils von dem extradiegetischen Erzähler erzählt. ${ }^{10}$ Diese Erzählinstanzen führen den Rezipienten allmählich in die fiktive Welt.

Theagenes und Charikleia werden von Piraten entführt und geraten in die Hände des Knemon, eines jungen Mannes aus Athen (Buch 1). Die Geschichte des Liebespaares erfährt Knemon erst nach dem Scheintod Charikleias, und zwar von dem ägyptischen Priester Kalasiris (Buch 2). ${ }^{11}$ Charikleia, Priesterin der Göttin Artemis im Delphi-Tempel, ist die Ziehtochter des Charikles, der ebenfalls Priester in Delphi ist. Sie lernt bei den Pythischen Spielen Theagenes, der von adliger Abstammung ist, kennen und verliebt sich in ihn (Buch 3). Charikleias Herkunft - sie ist ein Kind des äthiopischen Königspaars Hydaspes und Persinna - wird durch einen auf das Wickeltuch des Kindes geschriebenen Text enthüllt, und zwar durch den aus Memphis stammenden Priester Kalasiris, der die ägyptische Schrift entziffern kann. ${ }^{12}$ Auf Kalasiris' Vorschlag hin beschließen Theagenes und Charikleia, die Flucht zu ergreifen, weil das Mädchen dem Neffen des Charikles versprochen wurde (Buch 4).

Dann beginnt die lange ,Odyssee des Theagenes und der Charikleia nach Äthiopien, wobei sie diverse Hindernisse bewältigen müssen: Auf der Reise verlieren sie sich, weil sie von Seeräubern verfolgt werden (Buch 5). Charikleia und Kalasiris verkleiden sich als Bettler, um nach Theagenes zu suchen (Buch 6). Das Liebespaar

10 Zur Komposition der Aithiopika siehe Winkler 1982, 93-114; Morgan 1994, 100 - 109; Futre-Pinheiro 2014, 84-88. Futre-Pinheiro 2014, 86 hebt hervor: „Heliodorus plays with a limited number of characters who move in concentric circles. That is why we find a series of overlapping and interlocking stories. He masters the technique that consists of maintaining different lines of action in abeyance and using them at precisely the right moment to intensify the reader's interest and suspense.“ Der Fokus dieser Erzähltechnik, des Wechsels zwischen Wissen und Unwissenheit, liegt auf den Rezipienten, wie Winkler 1982, 95 anmerkt: „The focal point in this game of knowledge and ignorance must always be the reader, who is by turns puzzled and enlightened in a shifting chiaroscuro of irony, half-truths and recognitions.“ Zur Ambiguität der Aithiopika siehe Kruchió 2017, 188 -192.

11 Kalasiris fungiert als eine wichtige Erzählerfigur, die bisweilen die Rolle des allwissenden auktorialen Autors einnimmt. Zu dieser Romanfigur siehe Morgan 2003, 173, mit weiterer Literatur; FutrePinheiro 1991, 69 - 83, sowie Bierl 2007, 305-309, und Kruchió 2017, 177 f. Zum Verhältnis des Kalasiris zum Romanleser beobachtet Bierl 2007, 306: „Im ägyptischen Kleid des Weihepriesters involviert er [sc. Kalasiris] ihn [sc. den Leser] durch fremdartige und theatrale Verfahren in das Labyrinth der Erzählung. “ Kalasiris stellt als intradiegetischer Erzähler die Vorgeschichte in zwei Teilen dar (2,24-5,1 und 5,17-33). Zum Begriff des intradiegetischen Erzählers siehe Genette ${ }^{3} 2010$, 162f. Zur Bedeutung des Übergangs von der ersten zur zweiten Hälfte der Aithiopika (also vom Ende des Berichts des Kalasiris zur Geschichte über Knemon und Nausikleia) siehe Grethlein 2016, 316-335.

12 Anhand des Wickeltuchs erfährt man, warum Charikleia eine helle Hautfarbe hat: Im Moment der Empfängnis von Charikleia hatte Persinna auf ein Bild der unbekleideten weißhäutigen Andromeda geschaut, das es im Schlafzimmer gab. Dies soll das Aussehen des Kindes beeinflusst haben. Um nicht des Ehebruchs bezichtigt zu werden, beschließt Persinna, ihre Tochter aussetzen zu lassen, und gibt ihr Schmuck und das Wickeltuch mit. Ein äthiopischer Priester findet das Kind und vertraut es Charikles nach sieben Jahren an. Dazu Bettenworth 2013, 194 mit Anm. 1. Somit ergibt sich auch eine biologische Erklärung, warum Charikleia weißhäutig geboren ist (ebd. 199f.). Zur Empfängnis Charikleias siehe Reeve 1988, 81-112 sowie Olsen 2012, 301-322. 
trifft sich wieder in Memphis, wo Kalasiris den Tod findet, nachdem er seine beiden Söhne wiedergefunden hat. Eine weitere Rivalin begegnet dem Liebespaar, nämlich Arsake, die Frau des persischen Statthalters, die sich in Theagenes verliebt (Buch 7). Sie versucht vergeblich, mit Hilfe ihrer Dienerin Kybele Theagenes für sich zu gewinnen und Charikleia aus seinem Leben zu verbannen (Buch 8). Der Satrap Oroondates mit seinen persischen Truppen wird in der ägyptischen Stadt Syene durch den äthiopischen König Hydaspes angegriffen und besiegt (Buch 9). Hydaspes verschleppt Theagenes und Charikleia in die äthiopische Hauptstadt Meroë, um sie den Göttern als Menschenopfer bei der Siegesfeier darzubringen. Dann erkennt er seine Tochter wieder; der Roman endet mit der Aussicht auf eine Hochzeit des Paares in Äthiopien (Buch 10). ${ }^{13}$

\section{Theatralität in den Aithiopika}

Von Beginn an rückt Heliodor die Aithiopika durch einige bewusst gewählte Ausdrücke in die Nähe eines Bühnenstücks und bereitet so den Leser darauf vor, im Folgenden sein besonderes Augenmerk auf die Theatralität ${ }^{14}$ seines Romans zu richten. So deuten die Begriffe theatron und skene gewissermaßen auf das „erste Bühnenbild“ hin (1,1,6,f.), ${ }^{15}$ in dem ein Schiff und das Liebespaar präsentiert werden. Mit Hilfe sozusagen einer Kamera-Aufnahme wird eine Reihe von Einzelbildern vorge-

13 Das Liebespaar findet sein Happy End nicht an dem Ort, an dem ihre Geschichte begann (in Delphi), wie in den meisten früheren Romanen, sondern in der äthiopischen Stadt Meroë. Diese wird somit zum Schauplatz wichtiger Ereignisse (z.B. der Anagnorisis Charikleias durch ihre Eltern, der Beinahe-Opferung des Theagenes, der Hochzeit von Theagenes und Charikleia), aber eben erst am Ende des Romans. So auch Morgan 2012, 557. Vgl. auch De Temmerman 2014, 247: „Heliodorus’ novel, then, narrates the heroine's homecoming, or nostos. However, it subverts this central theme by staging not a classical return to Greece but a trenchant relocation of the heroes to the edges of the world instead.“ Ähnlich bereits bei Smith 2007, 227-234. Zur ,Identität” der Aithiopika siehe Whitmarsh 2011, 108-135 (bes. 128: „We can read Charicleia and Theagenes as the supplanting of a false, Hellenocentric, centre-periphery romance with a true, linear, Ethiopo-centric text“); vgl. auch Stephens 2008, 56-71 (bes. 70). Vom Zentrum, vom Omphalos, dem „Nabel der Welt“ (d.h. Delphi), wird der Fokus Heliodors auf die Peripherie und die Ränder (d.h. Meroë/Äthiopien) verlagert.

14 Chaniotis 1997, 222 definiert Theatralität folgendermaßen: „[...] the effort of individuals or groups to construct an image of themselves which is at least in part deceiving, because it either is in contrast to reality or because it exaggerates or partly distorts reality. [...] Furthermore, the effort to gain control over the emotions and the thoughts of others, to provoke specific reactions, such as sorrow, pity, anger, fear, admiration, or respect. To achieve these two aims, that is, to construct an illusion and to control the emotions and thoughts of others, a variety of means of verbal and non-verbal communication may be applied: a carefully composed text, a particular costume, images and mechanical devices, the selection of the space where the 'performance' takes place, the control of the voice - its volume, tone, stability, and flexibility, body language - pose, gestures, movement of the feet -, facial expressions, the choice of timing."

$15 \mathrm{Zu}$ den Theater-Begriffen, die bei Heliodor auftreten, siehe Walden 1984,1-43. Dazu auch Paulsen 1992, 22-28. 
führt, die nach und nach einen Gesamteindruck vermitteln. ${ }^{16}$ Zuschauer ( $\left.\theta \varepsilon \omega \rho o i ́\right)$ dieser „Theateraufführung“ sind die Räuber, die Theagenes und Charikleia entführen werden. ${ }^{17}$ Die Leser des Romans schauen gewissermaßen den Räubern über die Schulter, denn ihnen muss - genau wie den Räubern - die Szene zunächst unverständlich bleiben. ${ }^{18}$ Die Barbaren werden eingeladen, ein griechisches Theaterstück $\mathrm{zu}$ verstehen.

Die theatralische Atmosphäre der Aithiopika ist auch durch weitere Elemente gekennzeichnet, die die Theaterkultur der Kaiserzeit charakterisierte, beispielsweise das Klagen. Dieses rhetorische Mittel ist nicht untypisch für Heliodors Roman. Die Romanfiguren sind mit dieser Technik vertraut, wie es sich in ihren langen Klagemonologen erkennen lässt. ${ }^{19}$ Im Gegensatz zu den anderen Romanautoren verfügen Heliodors Klagepartien nicht nur über die typischen Merkmale einer Klagemonodie (Asyndeton, rhetorische Fragen, einfache Satzstruktur, Apostrophierungen, Vergleiche zwischen Vergangenheit und Gegenwart), sondern bieten auch Rekapitulationen und sogar Auskünfte über den weiteren Fortgang der Handlung. ${ }^{20}$

Weitere Allusionen auf die Tragödie sowie theatralische Hinweise enthält die Szene, in der Theagenes sein und Charikleias Leben ins literarische Genre der Tragödie verlagert (5,6,3f.): [...] „Er [sc. der Daimon oder die Gottheit] treibt solch ein feindseliges Spiel gegen uns, als führe er ein Theaterstück mit uns auf. Warum kürzen wir denn diese Tragödiendichtung nicht ab [...]?“. ${ }^{21}$ Die Gottheit, die das Schicksal des

16 Vgl. Telò 2011, 581-613. Bereits Paulsen 1992, 54. Dazu auch Bühler 1976, 177-185; er weist darauf hin, dass Heliodor „die Technik der modernen Filmkamera“ in Anspruch nehme und auf diese Weise „die Züge eines sensiblen Künstlers“ trage (ebd., 178). Zu den intermedialen Einflüssen, und zwar der Ikonographie der Eröffnungsszene mit ihren symposiastischen Elementen (Reste eines Festmahls und Trinkgefäße) siehe Tagliabue 2015, 445 - 468. Zur Ekphrasis in Heliodors Roman siehe Webb 2009, 178 183; vgl. auch Bartsch 1989 (bes. S. 77-79) sowie Goldhill 2007, 1-19. Grundlegend zur Bedeutung der visuellen Imaginationskraft für die Leserrezeption in fiktiven Werken Esrock 1994. Ausführlicher zu Heliodors Ekphrasis im Verhältnis zu seinen Vorläufern Homer und Herodot siehe Menze 2017.

17 Vgl. Heliod. 1,1,6f. Siehe auch Morgan 2012, 574 sowie Paulsen 1992, 54.

18 Hierzu Morgan 1991, 86: „The scene is presented exclusively from the bandits' point of view. Information about it is relayed to the reader only through their perceptions. The whole passage is structured much like a single cinematic camera-shot zooming in from panorama to close focus, moving from detail to detail. There is no intervention from an omniscient narrator to provide answers to the riddles posed by the scene [...]. “ Siehe dazu auch Morgan 1996, 442; Winkler 2002, 161-184 sowie FutrePinheiro 2014, $80 \mathrm{f}$.

19 Zum Klagen als rhetorisches Mittel im griechischen Roman siehe Birchall 1996, 1-17 (zu Heliodor 14-17); vgl. auch Webb 2007, 530 -533. Sie konstatiert: „The rhetorical use of language affects the readers emotionally and intellectually and draws them into the text, just as Cnemon is drawn into Calasiris' narration, so that the readers share, through logos, in the experience of eros that is at the centre of the narratives“ (ebd. 538).

20 Vgl. 1,8,2f.; 2,1,2f.; Birchall 1996, 14f. Ausführlich zu tragischen Klagemonologen bei den griechischen Dramatikern Aischylos, Sophokles und Euripides Schauer 2002.

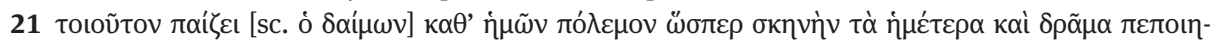

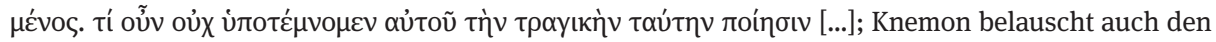
Klagemonolog der schönen Charikleia, die sich im Haus des Nausikles befindet und ebenfalls ihre 


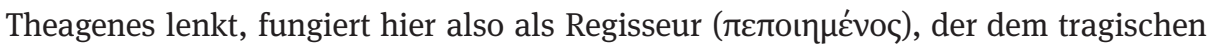
Helden Anweisungen gibt. ${ }^{22}$

Auch Charikleia ist sich ihrer Rolle als Akteurin in einem tragischen Stück bewusst: Im ersten Buch bittet sie ihre Entführer, sie zu töten, denn erst dann kann ihr Drama zu einem Ende kommen: „Erlöst uns (jetzt) von den uns widerfahrenen Leiden durch unsere Ermordung und macht damit unserer Tragödie ein Ende“ (1,3,1). ${ }^{23}$ Im sechsten Buch beklagt sie ihr tragisches Schicksal und beschuldigt die Götter, dass sie ein Happy End, nämlich die Wiedervereinigung mit Theagenes, hinauszögerten, um die dramatische Geschichte auf der Bühne zu verlängern: „So endlos habt ihr unsere Tragödie in die Länge gezogen, dass sie jedes andere Stück übertönt“ $(6,8,5){ }^{24}$

Das tragische Vokabular sowie die wörtliche und metaphorische Affinität zum Drama sind bedeutsam vor den Hintergrund der Popularität und stetig wachsenden Verbreitung der Theaterspektakel im römischen Reich, wodurch der jeweilige Kaiser versuchte, nicht nur das Publikum zu unterhalten, sondern auch seine politische Macht und Dominanz zutage treten $\mathrm{zu}$ lassen. ${ }^{25}$ Die dramatische Inszenierung des Beinahe-Todes der Charikleia - sie wird zum Tod auf dem Scheiterhaufen verurteilt, weil sie Kybeles Intrige zum Opfer gefallen ist $(8,9,10)$, aber dann doch nicht umgebracht - könnte auf die Hinrichtungen im Amphitheater anspielen, um das Ereignis dem Leser in aller Drastik vor Augen zu führen.

\section{Heliodors Umgang mit der Tragödie (Euripides)}

Heliodors Aithiopika zeichnen sich dadurch aus, dass sie eine Fülle von intertextuellen Bezügen und literarischen Anspielungen auf Gattungen enthalten, die dem ge-

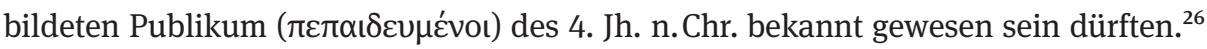
Dabei zeigen sich Bezüge nicht nur in Übereinstimmungen und Nachahmungen,

vergangenen Leiden aufzählt (5,2,7-10); der Auslöser dieser Übel sei der Dämon oder die Gottheit,

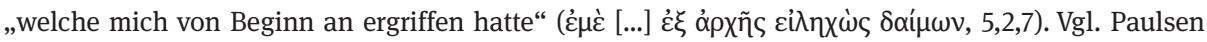
1992, 64 und $152 \mathrm{f}$.

22 Dazu siehe Paulsen 1992, 65.

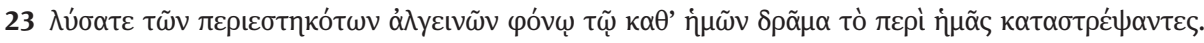
Dazu auch Paulsen 1992, 30 und 55.

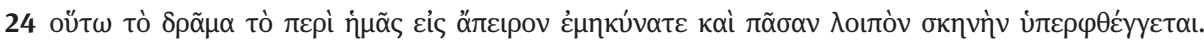
Paulsen 1992, 66.

25 Vgl. auch Connors 2008, 172f. Sie merkt richtig an: „From the novel's literal and metaphorical references to spectacle and to politics, key terms emerge as characteristic of each novel's distinctive ideas about the dynamics of civic life in an imperial world.“ (ebd. 173).

26 Wie Feuchtenhofer 2010, 7 richtig anmerkt: „Er [sc. Heliodor] schöpft aus dem für die Gattung typischen Motivpool und entspricht somit den Erwartungen seines Publikums.“ Zum literarischen gebildeten Umfeld des antiken Romans siehe Bowie 2008, 17- 38 (bes. 18-21). Zu den Rezipienten des antiken Romans siehe Hunter 2008, 261-271 sowie Konstan 2009, 1-17. Einführend zu den Vorläufern des Romans Fusillo 1991. Vgl. dazu Elmer 2008, 411- 429 sowie Stephens 2008, 65 f. 
sondern auch in gezielten Abweichungen von den Prätexten. In diesem Sinne stellte bereits Pletcher 1998 fest: „Heliodor was revealed not only as an author who has a masterful touch with plot and storytelling, but with creative reinterpretation and adaptation of his literary predecessors.“27

Ein Prätext für den Roman ist sicherlich die homerische Odyssee, eine Heimkehrund Reise-Erzählung mit besonderem Fokus auch auf der Liebesbeziehung zwischen Odysseus und Penelope nach vielen Gefährdungen und Intrigen. ${ }^{28}$ Der Vergleich zwischen Odyssee und Aithiopika lässt sich nicht nur anhand motivischer Parallelen, sondern auch aufgrund von Ähnlichkeiten in der Erzählstruktur nachweisen. ${ }^{29}$ Andere Gattungen wie die Komödie (z. B. Menander) sind ebenfalls einflussreich auf Heliodors Roman. ${ }^{30}$ Die Tragödie indes, und zwar die Stücke des Euripides, haben noch viel stärker auf ihn eingewirkt. ${ }^{31}$

Bereits Aristoteles bezeichnete Euripides als den „tragischsten“ ( aller Dichter (Poet. 13, 1453a29). In seinen Ausführungen über die handlungsstrukturellen Momente der Tragödie wie z.B. die Anagnorisis sieht Aristoteles Euripides' Iphigenie in Aulis als exemplarisch an. ${ }^{32}$ Die Anagnorisis ist zwar kein archetypisches Merkmal der euripideischen Tragödien, ${ }^{33}$ aber in Verbindung mit der Peripetie gehört sie zu den wichtigen Merkmalen der dramatischen Gattung.

Auch Heliodor greift die Anagnorisis auf und stellt eine Reihe von AnagnorisisSzenen dar, die allmählich zur Hauptanagnorisis (zwischen Charikleia und ihren Eltern) führen: Zunächst sehen sich Charikleia und ihr väterlicher Freund Kalasiris im

27 Pletcher 1998, 23.

28 Vgl. z. B. Keyes 1922, 42-51; Lalanne 2008, 121-132; Feuchtenhofer 2010, 53-71 mit Anm. 93.; Lefteratou 2017, 271-273. Zum ideologischen Einfluss Homers auf Heliodor siehe Whitmarsh 1998, 93124.

29 Vgl. oben S. 211 Anm. 11. Heliodor lässt sein Publikum in medias res einsteigen, den Faden zum Beginn der Romanhandlung findet man erst am Ende des 5. Buches. Diese narratologische Technik findet sich bereits in der Odyssee. Siehe dazu Feuchtenhofer 2010, 23 - 28 mit weiterer Literatur. Vgl. auch Morgan 1989, 99 -113; Anderson 1997, 304-309; Ziegler 2011, 2.

30 Einführend Corbato 1968, 5-44. Vgl. auch Brethes 2007 (bes. 103-124 und 163-186).

31 Siehe hierzu Marino 1990, 203-218, Morgan 2012, 574 sowie Doody 2013, 105-125.

32 Arist. Poet. 11, 1452b 5-10; 15, 1454a 31-33; 16, 1454b19-1455a20. Vgl. besonders 16, 1455a 16-20: „Aber die beste Anagnorisis von allen ist die, die sich aus den Ereignissen selbst ergibt, wenn die Überraschung durch das, was wahrscheinlich ist, entsteht wie im Ödipus des Sophokles und in der Iphigenie; denn es ist wahrscheinlich, dass sie einen Brief übergeben will. Solche Wiedererkennungsszenen sind nämlich die einzigen, die ohne erfundene Zeichen wie Halsbänder auskommen“

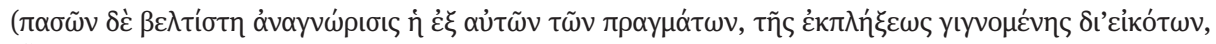

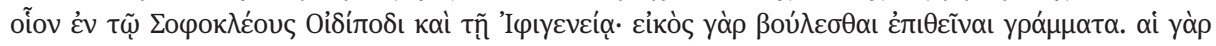

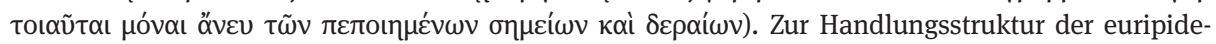
ischen Tragödien siehe Dubischar 2017, 367-389.

33 Das Motiv findet sich bereits bei Homer: vgl. die Wiederkennungsszenen mit Alkinoos, Telemachos, Eumaios und Eurykleia vor der krönenden und letzten Anagnorisis des Odysseus mit Penelope. Dazu siehe Lefteratou 2017, 183-185. Dazu auch Cave 1988, 40 - 45; zur Anagnorisis-Thematik siehe zuletzt Montiglio 2013. 
Haus des Nausikles wieder $(5,11,2) .{ }^{34}$ Erst nach vielen Hindernissen und weiteren Anagnorisisszenen - z. B. erkennt Kalasiris seine Söhne Thyamis und Petosiris vor den Stadtmauern von Memphis wieder (7,6,4-7,7,4), und Theagenes erkennt die als Bettlerin verkleidete Charikleia wieder (7,7,6f.) - kommt es zur abschließenden Anagnorisis von Charikleia und ihren Eltern (Buch 10). Die genannten Szenen tragen zur hohen Spannung des Romans bei und gelten als Bestandteile der Tragödie über Charikleia und Theagenes. Die Wiedervereinigung Charikleias mit ihren Eltern und der glückliche Ausgang ihrer Liebesgeschichte mit Theagenes werden schließlich als

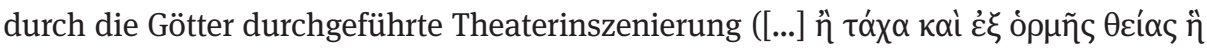

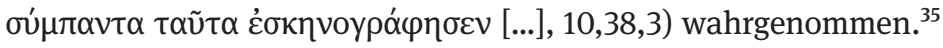

Während auch die späteren Dramen des Euripides durch die Anagnorisis den Kulminationspunkt der dramatischen Handlung erreichen (z. B. Ion und Helena), ${ }^{36}$ erweist sich bei Heliodor als Prätext für die entscheidende Wiedererkennung Charikleias mit ihren leiblichen Eltern und Beinahe-Opferung die Iphigenie in Aulis, um die Göttin Artemis zu versöhnen. ${ }^{37}$ Eine Parallele zu Heliodors Roman ergibt sich, wenn man berücksichtigt, dass Charikleia Priesterin der Artemis war - also genau derselben Gottheit, zu deren Besänftigung Iphigenie geopfert werden soll.

Neben der motivischen Nähe Heliodors zu Euripides (hier anhand der Konstruktion von Anagnoriseis) soll nun die Verarbeitung einiger mythologischen Stoffe bei Euripides und Heliodor in den Blick genommen werden. Der Hippolytos-PhaidraMythos ist eine prominente tragische Quelle, von der sich Heliodor zur Gestaltung seines ersten und siebten Buches hat anregen lassen. ${ }^{38}$ Bereits im ersten Buch erzählt

34 Paulsen 1992, 69 merkt richtig an: „Nausikles [...] fungiert bei diesem Anagnorismos als Publikum“, weil er noch nicht das Verhältnis zwischen Charikleia und Kalasiris erahnt hat.

35 Dazu Paulsen 1992, 79. Vgl. auch Lefteratou 2017, 91 f. Bei Heliodor ist es die Tochter Charikleia, die sich zu erkennen gibt, indem sie Hydaspes die Erkennungszeichen (Schmuckstücke und beschriftetes Band) vorweist; vgl. 10,12,1-3.

36 Zum Vergleich des euripideischen Ion mit Heliodor siehe Clavo 2003, 299-321.

37 Ausführlich zu den intertextuellen Bezügen zwischen Euripides' Tragödie Iphigenie in Aulis und Heliodors Roman siehe Lefteratou 2013, 200 -222, sowie Lefteratou 2017, 80-100. Das Motiv der Kindsopferung erinnert an die Phönissen des Euripides, in denen Kreon zu Ares’ Versöhnung seinen Sohn Menoikeus opfern soll. Dieser aber möchte dem Gott nicht Folge leisten (Phoen. 919, 963-966). Menoikeus tötet sich dennoch freiwillig aus Heimatliebe. Bei Heliodor hingegen beharrt Hydaspes darauf, obwohl er seine Tochter erkennt, sie zu opfern, weil er dem Volk dieses ,Spektakel` auf der Bühne bieten und den Göttern das Menschenopfer darbringen möchte (10,16,3-10,17,3). Erst als das gesamte Volk Einspruch legt, rückt Hydaspes von seiner Entscheidung ab. Nichtsdestrotz möchte er Theagenes' Opferung weiterhin ausführen. Dann bittet ihn Charikleia, ihr die Tötung ihres Geliebten zu überlassen, weil sie sich mit dem Opfermesser ebenfalls das Leben nehmen möchte $(10,20,2)$. Diese Selbstlosigkeit der Charikleia erinnert an andere opferbereite Frauen, wie wir sie auch in Euripides' Tragödien finden: z. B. Alkestis im gleichnamigen Drama (für die Rettung ihres Mannes Admetos). Vgl. Matthiessen 2004, 45-48.

38 Vgl. Cueva 2004, 87-90. Ein Beispiel für diese Rezeption ist die doppelte Intrige Demainetes gegen Knemon: zuerst durch Demainete allein $(1,10,3 \mathrm{f}$.) und zweitens mit Hilfe ihrer Dienerin Thisbe $(1,11,2)$. 
der junge Athener Knemon, dass sich seine Stiefmutter Demainete in ihn verliebt

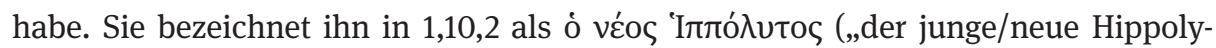
tos“). ${ }^{39}$ Als sie jedoch von ihm zurückgewiesen worden sei, habe sie ihn durch böse Intrigen mit Hilfe ihrer Sklavin Thisbe aus dem Haus seines Vaters vertrieben (1,9,1-1,18,5). Im Bekränzten Hippolytos des Euripides (das zweite Hippolytos-Stück; das erste ist verloren) beschließt Phaidra nach Hippolytos' Ablehnung, einen Brief für Theseus zu hinterlassen, in dem sie ihren Stiefsohn beschuldigt.

Im siebten Buch der Aithiopika muss sich der Protagonist Theagenes der Annäherungsversuche Arsakes, der Frau des persischen Statthalters, erwehren, die schließlich mit Rache droht (7,2,1-4). Ähnlich wie im Bekränzten Hippolytos gibt es hier eine alte Dienerin, die eine Vermittlerrolle übernimmt. ${ }^{40}$ Des Weiteren führt die Theagenes-Arsake-Episode zu gravierenden Konsequenzen für Arsake, denn sie erhängt sich - wie die euripideische Phaidra. ${ }^{41}$

Heliodor rekurriert in seinen Aithiopika auch auf die euripideischen Phönissen, die aufgrund ihrer Aufnahme in den Schulkanon wachsende Beliebtheit und hohes Ansehen in der Kaiserzeit genossen: ${ }^{42}$ Thyamis, der ältere Sohn des Kalasiris, wird wie Polyneikes von seinem jüngeren Bruder Petosiris, vergleichbar dem Eteokles, eigenmächtig aus seinem Priesteramt in Memphis vertrieben. ${ }^{43}$ Daraufhin beschließt Thyamis, gegen seinen Bruder und sein eigenes Heimatland zu kämpfen. In beiden Brüder-Konstellationen geht es um die ordnungsgemäße Wiederherstellung der Machtposition. Während bei Heliodor Kalasiris rechtzeitig ankommt, um als deus ex machina ins Geschehen einzugreifen und den Streit seiner Söhne zu schlichten (He-

Siehe dazu auch Lefteratou 2017, 157-170. Lefteratou 2017, 168 beobachtet: „Cnemon [...] presents his tale through a dramatic lens, emphasising the ties of his own stepmother's plot with his tragic models.“ 39 Der Textausgabe von Lumb und Rattenbury $(1935,15)$ zufolge weist die genannte Textstelle eine lacuna auf. Anders bei Colonna 1938, 14. Auch wenn die Textstelle korrupt ist, lässt sich vermuten, dass Heliodor den Hippolytos-Mythos im Allgemeinen aufgreift. Darauf nehmen auch andere Romanautoren Bezug, und zwar Chariton (Chaireas) und Xenophon (Habrokomes); vgl. De Temmerman 2014, 47-50 und 142-145.

40 Vgl. Rocca 1976, 25-31.

41 Vgl. Heliod. 8,15,2: „Arsake ist gestorben, nachdem sie eine Schlinge aufgeknüpfte hatte, um sich

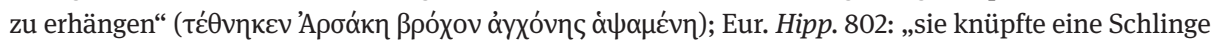

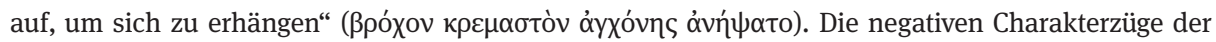
Demainete und Arsake, die als Gegenfiguren zu Charikleia fungieren, wurden bereits von Morgan 1989, 99-113, herausgearbeitet. Vgl. auch Smith 2007, 228-230. Zu doppelten Zugriffen auf denselben Mythos bei Heliodor siehe Morgan 1998, 60 - 78.

42 Hierzu siehe Matthiessen 2002, 210 und Cribiore 2001b, 241-259. Der Konflikt der Ödipus-Söhne findet sich auch in weiteren literarischen tragischen Bearbeitungen, z. B. in den Sieben gegen Theben des Aischylos, in der Antigone sowie im König Ödipus des Sophokles. Nichtsdestoweniger weist Heliodors Roman mehr Anspielungen auf die Phönissen des Euripides als auf andere Tragödien auf, insbesondere in Bezug auf die ausführlichen Schilderungen des Bruderkampfs und die Vermittlerrolle des Kalasiris (zusammen mit Charikleia; vgl. Iokaste und Antigone in den Phönissen). Dazu siehe auch Whitmarsh 2011, 112f. mit Anm. 31 (und weiteren Literaturhinweisen).

43 Heliod. 1,33,2; vgl. auch 7,2,2; 7,8,7. Dazu auch Paulsen 1992, $156 \mathrm{f}$. 
liod. 7,6,4-7,7,4), erscheinen Iokaste und Antigone in den Phönissen des Euripides auf dem Kampfplatz zu spät. ${ }^{44}$ Die Brüder töten sich gegenseitig (1264-1282; 1429-1454), wohingegen Kalasiris’ Vermittlungsversuche zur Versöhnung seiner Kinder beitragen (Heliod. 7,7,3; 7,8,4f.; 7,8,7).

In Kalasiris spiegelt sich aber nicht nur Iokaste, die Mutter der kämpfenden Brüder, sondern auch ihr Vater, Ödipus. Der Priester erfüllt aufgrund des Todes seiner Frau die doppelte Funktion des Vaters und der Mutter für seine Söhne. ${ }^{45}$ Betrachtet man das Ergebnis, so erscheint die Konstellation in Heliodors Roman absolut gegensätzlich zu derjenigen im thebanischen Sagenkreis: Dem Tod der Söhne und dem fragwürdigen Ende des Ödipus steht hier eine glücklich wiederhergestellte Familienidylle gegenüber. Obwohl beide den Entschluss fassen, ihre Heimat zu verlassen, um den schrecklichen Prophezeiungen zu entgehen, kehrt nur Kalasiris heim und rettet seine Söhne vor dem gegenseitigen Mord. Doch genau darin liegt der Schlüssel zum harmonischen Ende. Kalasiris wird gewissermaßen zum positiven Anti-Ödipus, weil er den Umschlag des Unglücks in Glück bewirkt.

Es lässt sich also festhalten, dass es in den Aithiopika ein dichtes Netz intertextueller Bezüge zu den Euripides-Tragödien gibt; Heliodor greift verschiedene mythologische Sagenstoffe (hier z. B. Phaidra, Iphigenie und Ödipus) auf, die bei Euripides vorkommen, und stellt so Bezüge zum Handlungsverlauf, zu strukturellen Elementen und zu Handlungsträgern der Tragödie her, und zwar nicht nur in Anlehnung an diese, sondern auch mittels Kontrastierung. Einige Handlungselemente und Figuren werden verändert und umgestaltet, und dies in einer Weise, die ganz deutlich Heliodors eigene Handschrift trägt (wie etwa Ödipus in Kalasiris oder die zu den Phönissen entgegengesetzte Figurenkostellation). So gewinnt der Roman nicht nur eine theatralische Dimension, sondern grenzt sich auch als eigene literarische Gattung gegenüber der Tragödie ab. Der produktive Umgang mit dem Tragödienprätext begründet den Innovationscharakter des Romans.

44 Vgl. Bretzigheimer 1999, 69 und Paulsen 1992, 168 f. und 262 (Anm. 90).

45 Vgl. Heliod. 2,23,2, wo Kalasiris Knemon von seinen väterlichen Gefühlen für Theagenes und Charikleia erzählt: „O Fremder, sagte [sc. Kalasiris], sie [sc. Theagenes und Charikleia] sind mir Kinder geworden, ohne dass eine Mutter sie mir geschenkt hätte; durch eine glückliche Fügung haben die Götter sie mir nämlich zu solchen gemacht, und die Schmerzen meines Herzens haben sie geboren, und meine Einstellung zu ihnen hielt man für die zu leiblichen Kindern, und aufgrunddessen hielten

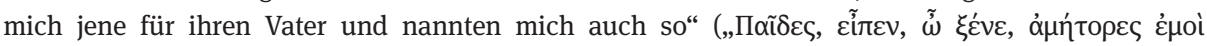

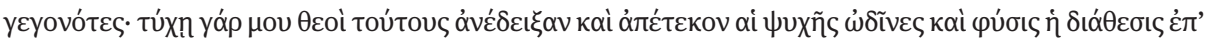

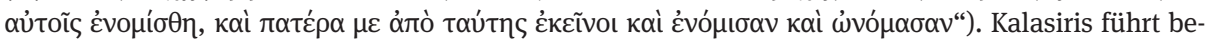
sonders die „Schmerzen“ ( $\dot{\omega} \delta$ Ĩvعৎ) seines Herzens an, wörtlich die ,Wehen“ seines Herzens, die sie ihm „geboren“ hätten, und begibt sich somit in den mütterlichen Bereich (vgl. auch Heliod. 2,22,4). Nach dem Tod des Kalasiris schreibt auch Charikleia diesem, obwohl Charikles sie aufgezogen hatte, die väterliche Identität sowie die Rolle ihres Beschützers und Retters zu (7,14,6): „Aber sieh mal, ich werde dir [sc. Kalasiris], Ernäher und Retter, auch ,Vater' hinzufügen, auch wenn der Dämon [sc. die Gottheit]

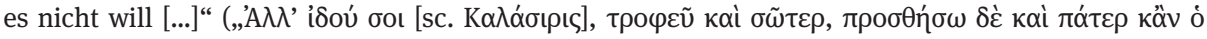

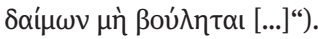




\section{Heliodor und der, fragmentarische ${ }^{e}$ Euripides}

Im Folgenden soll noch der Frage nachgegangen werden, ob und inwieweit auch andere euripideische Tragödien, die uns heute nur im fragmentarischen Überlieferungszustand vorliegen, hinsichtlich Thematik und Motivik in den Aithiopika aufgerufen sein könnten. Die Rekonstruktion der im Folgenden behandelten Tragödienstoffe erfolgt aus den Fragmenten und aus der indirekten Überlieferung (z.B. Scholien, Anthologien).

Auf die Nachricht von Kalasiris' Tod rät ein Tempeldiener Theagenes und Charikleia dazu, nicht mit Tränen, sondern mit Freude Abschied von Kalasiris zu nehmen $(7,11,9)$. Diese Passage enthält ein direktes Zitat aus der euripideischen Tragödie Kresphontes: ${ }^{46}$ „[Wir sollten die Toten] freudig und lobpreisend aus dem Haus gelei-

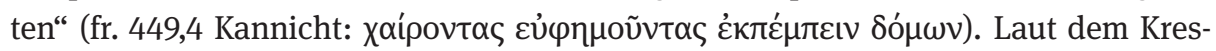
phontes-Mythos ermordet Polyphontes Kresphontes, den Herrscher Messeniens, und heiratet dessen Witwe Merope. Ihr Sohn, der bei Euripides ebenfalls Kresphontes heißt, wird vor Polyphontes' Nachstellungen gerettet und kehrt als Erwachsener zurück, um sich an seinen Vater zu rächen. ${ }^{47}$ Er gibt sich nicht zu erkennen und erzählt Polyphontes, dass er den (vermeintlichen) letzten Nachkommen des Kresphontes getötet habe. Das Zitat gehört vermutlich zu der Szene, in der Merope Polyphontes davon überzeugen möchte, dass sie den Tod ihres (vermeintlich verstorbenen) Sohnes erdulden könne. Denn sie wisse, dass ihr Sohn nun durch seinen Tod von Leiden

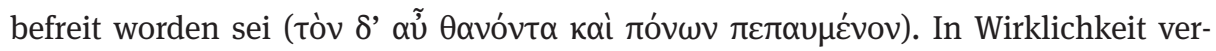
bündet sie sich mit ihrem wiederkannten Sohn Kresphontes, um gemeinsam Rache an Polyphontes zu nehmen.

Heliodor hatte vermutlich durch eigene Lektüre oder vermittelte, indirekte Rezeption Zugriff auf dieses Fragment aus den Kresphontes, das in der Antike vielfach rezipiert wurde, ${ }^{48}$ denn der Topos „Freude über den Tod“ war sehr verbreitet. Merope freut sich vorgeblich darüber, dass ihr Sohn durch seinen vermeintlichen Tod von Leiden befreit sei. Charikleia und Theagenes haben mit Kalasiris einen Menschen verloren, den sie mochten. Sie sollen sich freuen, dass sie ihn kennenlernen durften und dass er in eine bessere Welt gegangen ist $(7,11,9)$. Das euripideische Zitat wird also umgedeutet, was den kreativen Umgang Heliodors mit Euripides selbst (für den Fall einer eigenen Lektüre) oder mit der indirekten Überlieferung deutlich werden lässt.

Die Knemon-Demainete-Episode bei Heliodor scheint Parallelen nicht nur zum Bekränzten Hippolytos des Euripides aufzuweisen, sondern auch zu seinem fragmentarisch erhaltenen Hippolytos Kalyptomenos: In diesem Stück gesteht Phaidra

46 Die Erstaufführung der Tragödie ist wohl zwischen 430 und 424 v. Chr. zu datieren; vgl. Harder 1985, $3 \mathrm{f}$.

47 Zu diesem Fragment siehe Collard u. Cropp 2008, 506f.; Harder 1985, 24, 26, 92-98; Carrara 2014/ 2015, 3 mit Anm. 6-8.

48 Zur Überlieferung und Rezeption der euripideischen Tragödien siehe Mastronarde 2017, 11-26. 
ihrem Stiefsohn persönlich ihre Liebe (anhand der Rückverweise auf Senecas Phaedra); im Bekränzten Hippolytos enthüllt hingegen ihre Amme Phaidras Gefühle. ${ }^{49}$ Bei Heliodor verhält sich Demainete ähnlich und nähert sich ihrem Stiefsohn Knemon auf zudringliche Weise $(1,9,3)$. Während die Handlungen Knemons, Demainetes und Thisbes bei Heliodor in Einklang mit der Zurückweisung Phaidras durch Hippolytos und mit den Intrigen ihrer Amme (zusammen mit Phaidras Anklage) in Euripides' zweitem Hippolytos-Drama stehen, deutet die Zudringlichkeit Demainetes gegenüber Knemon auf Einflüsse der euripideischen Phaidra im ersten (verlorenen) HippolytosStück hin. ${ }^{50}$

Eine weitere euripideische Tragödie, auf die Heliodor rekurriert, ist die Andromeda. Anhand der Fragmente (114-156) lässt sich vermuten, dass dieses Stück mit Andromedas Klagemonodie und dem personifizierten Echo beginnt, das auf die Klage des Mädchens reagiert. ${ }^{51}$ Nach dem Auftritt des Chores kommt Perseus fliegend (als deus ex machina) nach Äthiopien und trägt das Haupt der Gorgone Medusa. Perseus verliebt sich auf den ersten Blick in Andromeda, die auf einem Felsen angekettet ist, wo sie zur Besänftigung Poseidons einem Ungeheuer zum Fraß ausgesetzt sein soll, und vergleicht sie mit einer Statue. Er möchte sie vor dem Ungeheuer retten und berichtet von seinen Abenteuern. ${ }^{52}$

Es finden sich bei Heliodor zwar keine direkten Zitate aus der euripideischen Andromeda, aber einige Anspielungen lassen sich erkennen: Heliodor führt Charikleias Abstammung auf Andromedas Geschlecht zurück. ${ }^{53}$ Das exotische Ambiente ist in beiden Werken präsent. Sowohl Charikleia als auch Andromeda sind beinahe ge-

49 Vgl. dazu Gill 22008, 166-172.

50 Vgl. TrGF 5, frr. 430, 432, 433, 434 Kannicht; test. i und iib. Zur Handlungsrekonstruktion des Hippolytos Kalyptomenos siehe Collard u. Cropp 2008, 466-471; vgl. auch Barrett 1964, 10-45: Er charakterisiert Phaidra im ersten Stück als ,shameless and unprincipled woman who when she fell in love with Hippolytos made a deliberate attempt to seduce him“ (ebd.11). Dieses Bild Phaidras unterscheidet sich von dem im erhaltenen Hippolytos-Stück; vgl. dazu Ebbott 2017, 116: „The possibility of multiple and changing reactions is a mark of Euripides' success in creating characters who can move us as much (even if in different ways) as they did the original audience." Das Liebesverlangen Phaidras wird auch im fragmentarischen gleichnamigen Stück des Sophokles behandelt (TrGF 4, frr. 677-693 Radt); vgl. dazu auch Sommerstein 2006, 248-317. In Sophokles' Phaidra sowie in Euripides' Hippolytos wird die verbotene Liebe Phaidras zu Hippolytos als „ein von den Göttern gesendetes Übel“ bezeichnet: So-

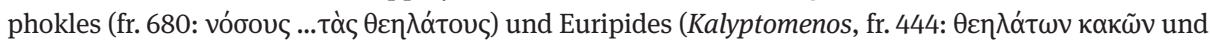

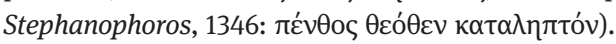

51 Zum Handlungsverlauf der Tragödie siehe Collard u. Cropp 2008, 124-155. Vgl. auch Klimek-Winter 1993.

52 Nicht aus der Fragmentenüberlieferung, aber aus der Hypothesis der Tragödie lässt sich entnehmen, dass Andromedas Eltern, die König von Äthiopien, nachdem Perseus das Ungeheuer getötet und Andromeda gerettet hat, ihr Einverständnis zu einer Ehe zwischen Andromeda und Perseus nicht geben wollen. Deshalb beschließt sie, mit ihrem Geliebten nach Argos zu fliehen. Am Schluss greift Athena ein und macht Andromeda zu einem Gestirn (Eur. Andr. test. iiia Kannicht). Dazu siehe auch Klimek-Winter 1993, 100.

53 Vgl. Heliod. 4,8,3. 
opfert worden (nach dem Auftrag ihrer Väter: Heliod. 10, 16f. und Eur. Andr. test. iiia; fr. 120 Kannicht). Darüber hinaus verliebt sich Perseus auf den ersten Blick in Andromeda und vergleicht sie mit einer Statue, also mit einem Kunstwerk (fr. 125). Charikleia stellt sich bei Heliodor als das Ebenbild Andromedas dar; ihre Mutter hat das Andromeda-Gemälde während des Beischlafs mit Hydaspes angeschaut, deshalb ist Charikleia weißhäutig geboren. ${ }^{54}$

Andromedas Porträt spielt eine wichtige Rolle im Roman; mit ihm beginnt und endet die Handlung in Form einer Ringkomposition: Der Anblick des Gemäldes durch Persinna und die Angst vor dem Vorwurf des Ehebruchs führen zur Entscheidung, Charikleia auszusetzen. Für die finale Anagnorisis wird Andromedas Porträt als Beweis für Charikleias Identität vorgeführt. ${ }^{55}$ Es lässt sich also klar eine Einwirkung des Kunstwerks auf das Schicksal der Charikleia erkennen. ${ }^{56}$

Außerdem kann man davon ausgehen, dass nicht nur die euripideische Andromeda, sondern auch verschiedene bildliche Zeugnisse des Andromeda-Mythos Einfluss auf Heliodor genommen haben dürften. ${ }^{57}$ Zudem könnten auch die Mimus- und Pantomimus-Darbietungen des Mythos Heliodor als Quelle gedient haben, welche in der Kaiserzeit sehr populär waren. ${ }^{58}$

Bei einem Vergleich Heliodors mit dem ,fragmentarischen` Euripides lassen sich einige thematische und motivische Ähnlichkeiten feststellen. Daher kann man davon ausgehen, dass über den aus dem im Schulunterricht gelesenen Kanon hinaus auch attische Tragödien rezipiert wurden, die dem Kanon nicht angehörten (und uns heute gar nicht oder nur im fragmentarischen Überlieferungszustand vorliegen). Plausibel ist auch der Einfluss der bildenden Kunst und pantomimischer Aufführungen auf Heliodors Roman, der in einer Zeit entstand, da sich solche Spektakel stetig wachsender Beliebtheit erfreuten.

\section{Schlussfolgerungen}

Durch die bewusste Anlehnung an Euripides' Tragödien versucht Heliodor, die Grenzen zwischen narrativ-epischer und dramatischer Gattung aufzuheben. Daher

54 Vgl. oben S. 207 Anm. 12.

55 Vgl. Anderson 1997, 318f.

56 Vgl. dazu Zeitlin 2013, 66 und 73-84. Vgl. den Ring mit Chaireas' Abbild, das Kallirhoe bei sich tragen möchte (1,14,9f.; 2,11,1-3). In der falschen Annahme, dass Chaireas tot sei, veranstaltet Kallirhoe ein großes Begräbnis für ihn und trägt ein Porträt von ihm als Replik des Originals auf dem Ring $(4,1,10)$. Ähnlich in der Szene, in der Chaireas das goldene Standbild Kallirhoes betrachtet, das im Tempel neben dem der Göttin Aphrodite steht (3,6,3f.).

57 Zur Rezeption des Andromeda-Mythos in visuellen Abbildungen siehe Klimek-Winter 1993, 108 116.

58 Einführend siehe Klein 2004, 155-174. Zum Spielwesen in der römischen Spätantike siehe zuletzt Puk 2014. Ein Beispiel für die Adaption einer Tragödie an die Bedürfnisse des Publikums durch Mimus und Pantomimus ist die Iphigenie bei den Taurern; dazu Hall 2010, 225-250. 
erhält die Geschichte von Charikleia und Theagenes tragische Züge, die allerdings mit einem Happy End kombiniert werden, und auch seinen Nebenfiguren verleiht Heliodor tragische Züge. Die Romanfiguren erinnern dabei an bekannte mythische Gestalten bzw. an deren Darstellung in den Tragödien. Hierdurch wird beim Rezipienten der Eindruck verstärkt, der ihm bereits in der ersten Szene vermittelt wurde: Er ist nicht nur Leser eines Buches, sondern gleichsam Zuschauer im Theater. So erhält der Roman eine visuelle Qualität, wie sie eigentlich den Theaterinszenierungen, der bildenden Kunst sowie den Arenaspielen zugehören.

Was speziell den Umgang Heliodors mit Euripides betrifft, ergibt sich, dass er zum einen mittels Konstrastimitationen und Subversionen seinen Figuren andere Züge verleiht, die von der Darstellungsweise des Euripides abweichen. Zum anderen kann man festhalten, dass auch die uns fragmentarisch erhaltenen Tragödien Einfluss auf die Aithiopika genommen haben (zumindest in der Form von thematischen und motivischen Allusionen). Euripides war für Heliodor und sein Publikum des 4. Jh. n. Chr. also nicht nur der Dichter des Hippolytos und der Phönissen, sondern auch anderer Tragödien, die uns nicht vollständig erhalten geblieben sind. Dass Euripides' literarische Bearbeitungen mythologischer Stoffe (durch direkte oder indirekte Überlieferungswege) für Heliodor eine wichtige Inspirationsquelle für sämtliche Handlungspatterns seines Romans waren, ist nun allerdings nicht vor allem Ausweis seines eigenen literarischen Geschmacks, sondern vielmehr ein weiteres Indiz für das allgemeine intellektuelle Milieu der Kaiserzeit, das Euripides als Literaten überaus schätzte und das Heliodor mit seinen Euripides-Reminiszenzen ansprechen wollte. Somit kann auch Heliodors Roman als eine wichtige Quelle für die Euripides-Rezeption im 4. Jh. n.Chr. angesehen werden.

\section{Literatur:}

\section{Textausgaben, Übersetzungen und Kommentare:}

Barrett 1964: William Spencer Barrett (Hg.), Euripides Hippolytos. Edited with Introduction and Commentary, Oxford.

Collard u. Cropp 2008: Christopher Collard u. Martin Cropp (Hgg.), Euripides. Fragments.

Aegeus-Meleager. Edited with Translation, Cambridge, M.A./London.

Colonna 1938: Aristides Colonna (Hg.), Heliodori Aethiopica, Rom.

Diggle 1994: James Diggle (Hg.), Euripidis Fabulae (3 Bände), Oxford.

Harder 1985: Annette Harder, Euripides' Kresphontes and Archelaos. Introduction, Text and

Commentary (Mnemosyne Suppl. 87), Leiden.

Kannicht 2004: Richard Kannicht (Hg.), Tragicorum Graecorum Fragmenta. Euripides (Bd. 5.1),

Berlin.

Kassel 1965: Rudolph Kassel (Hg.), Aristotelis de arte poetica liber, Oxford (Nachdruck 2002).

Klimek-Winter 1993: Rainer Klimek-Winter (Hg.), Andromedatragödien. Sophokles, Euripides, Livius Andronikos, Ennius, Accius. Text, Einleitung und Kommentar (BzA 21), Stuttgart.

Lloyd-Jones u. Wilson 1990: Hugh Lloyd-Jones u. Nigel G. Wilson (Hgg.), Sophoclis fabulae, Oxford (Nachdruck 1992). 
Lumb, Maillon u. Rattenbury 21960: Thomas Wallace Lumb, Jean Maillon u. Robert Mantle

Rattenbury (Hgg.), Heliodor. Les Éthiopiques (3 Bände), Paris.

Radt 1977 : Stefan Radt, Tragicorum Graecorum Fragmenta. Sophocles, Bd. 4, Göttingen.

Reardon 2004: Bryan P. Reardon (Hg.), Chariton Aphrodisiensis: De Callirhoe narrationes amatoriae, Leipzig.

Reymer 2001: Rudolf Reymer, Heliodor. Die Abenteuer der schönen Charikleia (Übersetzung mit einem Nachwort von Niklas Holzberg), Düsseldorf/Zürich.

Sommerstein 2006: Alan H. Sommerstein, Sophocles. Selected Fragmentary Plays I, Oxford.

Ziegler 2011: Sieglinde Ziegler, Heliodor, Aithiopika IV 1-12. Ein philologischer Kommentar, Frankfurt a. M.

\section{Sekundärliteratur:}

Anderson 1997: Michael J. Anderson, „The $\Sigma \Omega \Phi P O \Sigma Y N H$ of Persinna and the Romantic Strategy of Heliodorus'Aethiopica“, Classical Philology 92, 303-322.

Bartsch 1989: Shadi Bartsch, Decoding the Ancient Novel: the Reader and the Role of Description in Heliodorus and Achilles Tatius, Princeton.

Baumbach 1997: Manuel Baumbach, „Die Meroë-Episode in Heliodors Aithiopika“, Rheinisches Museum für Philologie 140, 333-341.

Bierl 2007: Anton Bierl, „Mysterien der Liebe und die Initiation Jugendlicher. Literatur und Religion im griechischen Roman“, in: Anton Bierl, Rebecca Lämmle u. Katharina Wesselmann (Hgg.), Literatur und Religion 2. Wege zu einer mythisch-rituellen Poetik bei den Griechen, Berlin/New York, 239-334.

Billault 1998: Alain Billault, „Les romanciers grecs et la tragédie“, in: Jean Leclant u. Jacques Jouanna (Hgg.), Le théâtre grec antique: la tragédie (Actes du 8ème colloque de la Villa Kérylos à Beaulieu-sur-Mer les 3 \& 4 octobre 1997), Paris, 179-194.

Birchall 1996: John Birchall, „The Lament as a Rhetorical Feature in the Greek Novel“, Groningen Colloquia on the Novel 7, 1-17.

Bettenworth 2013: Anja Bettenworth, „Die doppelte Andromeda. Eine umstrittene Wiederholung in Heliodors Aithiopika und ihr Einfluss auf die Deutung des Romans“, Rheinisches Museum für Philologie 156, 194-211.

Bowie 2008: Ewen Bowie, „Literary milieux“, in: Tim Whitmarsh (Hg.), The Cambridge Companion to the Greek and Roman Novel, Cambridge, 17-38.

Brethes 2007: Romain Brethes, De l'idéalisme au réalisme: une étude du comique dans le roman grec. Cardo 6, Salerno.

Bretzigheimer 1999: Gerlinde Bretzigheimer, „Brudermord und Kindesmord. Pseudotragik in Heliodors Äthiopika (mit einem Appendix zum Beginn des Romans)“, Wiener Studien 112, 59-86.

Bühler 1976: Winfried Bühler, „Das Element des Visuellen in der Eingangsszene von Heliodors Aithiopika“, Wiener Studien 10, 177-185.

Carrara 2009: Paolo Carrara, Il testo di Euripide nell' antiquità: Ricerche sulla tradizione testuale euripidea antica (sec IV a. C.-sec. VIII d. C.), Florenz.

Carrara 2014/15: Laura Carrara, „Die Trauer in Gaza. Zum Kontext der Fragmente inc. sed. 15 und 1 Amato von Prokop von Gaza“, Revue des Études Tardo-antiques 4, 1-10.

Cave 1988: Terence Cave, Recognitions. A Study in Poetics, Oxford.

Cerulli 2012: Enrico Cerulli, „Perspectives on the History of Ethiopia“, in: Alessandro Bausi (Hg.), Languages and Cultures of Eastern Christianity: Ethiopian (The Worlds of Eastern Christianity, 300-1500 Bd. 4), London/New York, 1-26. 
Chaniotis 1997: Angelos Chaniotis, „Theatricality Beyond the Theater. Staging Public Life in the Hellenistic World“, Pallas: Revue d'études antiques 47, 219-259.

Chuvin 1990: Pierre Chuvin, „La date des Éthiopiques d’Héliodore“, in: Pierre Chuvin (Hg.), Chronique des derniers païens: La disparition du paganisme dans l'Empire romain, du règne de Constantin à celui de Justinien, Paris 321-325.

Clavo 2003: Maria T. Clavo, „Comunicare a Delfi. Ione euripideo e le Etiopiche di Eliodoro“, in: Marcella Guglielmo u. Edoardo Bona (Hgg.), Forme di comunicazione nel mondo antico e metamorfosi del mito: dal teatro al romanzo (Culture Antiche. Studi e testi 17), Alessandria, $299-321$.

Connors 2008: Catherine Connors, „Politics and Spectacles“, in: Tim Whitmarsh (Hg.), The Cambridge Companion to the Greek and Roman Novel, Cambridge, 162-181.

Corbato 1968: Carlo Corbato, „Da Menandro a Caritone: studi sulla genesi del romanzo greco e i suoi rapporti con la commedia nuova“, Quaderni triestini sul teatro antico 1, 5-44.

Cribiore 2001a: Raffaella Cribiore, Gymnastics of the Mind. Greek Education in Hellenistic and Roman Egypt, Princeton.

Cribiore 2001b: Raffaella Cribiore, „The Grammarian's Choice: The Popularity of Euripides' Phoenissae in Hellenistic and Roman Education“, in: Yun Lee Too (Hg.), Education in Greek and Roman Antiquity, Leiden, 241-259.

Cueva 2004: Edmund P. Cueva, The Myths of Fiction: Studies in the Canonical Greek Novels, Ann Arbor.

De Temmerman 2014: Koen De Temmerman, Crafting Characters. Heroes and Heroines in the Ancient Greek Novel, Oxford.

Doody 2013: Margaret Doody, „Comedy in Heliodoros’ Aithiopika“, in: Michael Paschalis u. Stelios Panayotakis (Hgg.), The Construction of the Real and the Ideal in the Ancient Novel, Groningen, $105-125$.

Dubischar 2017: Markus Dubischar, „Form and Structure“, in: Laura McClure (Hg.), A Companion to Euripides, Malden, MA/Oxford, 367-389.

Ebbott 2017: Mary Ebbott, „Hippolytus“, in: Laura McClure (Hg.), A Companion to Euripides, Malden, MA/Oxford, 107-121.

Elmer 2008: David F. Elmer, „Heliodoros’s ,Sources‘: Intertextuality, Paternity, and the Nile River in the Aithiopika“, Transactions of the American Philological Association 138, 411-450.

Esrock 1994: Ellen J. Esrock, The Reader's Eye: Visual Imaging as Reader Response, Baltimore.

Ferrini 1990: Maria Fernanda Ferrini, „Le parole e il personaggio: Monologhi nel romanzo greco“, Giornale italiano di filologia 42, 45-85.

Feuchtenhofer 2010: Bettina Feuchtenhofer, Gleichzeitigkeit als Erzählstrategie in Heliodors Aithiopika, Wien.

Fusillo 1991: Massimo Fusillo, Naissance du roman (übs. von M. Abrioux aus dem Italienischen: /l romanzo greco. Polifonia ed Eros, Venedig 1989), Paris.

Futre-Pinheiro 1991: Marília P. Futre-Pinheiro, „Calasiris’ Story and its Narrative Significance in Heliodorus' Aethiopica“, in: Heinz Hofmann (Hg.), Groningen Colloquia on the Novel, Bd. 4, Groningen, 69-83.

Futre-Pinheiro 2014: Marília P. Futre-Pinheiro, „Heliodorus, the Ethiopian Story“, in: Edmund P. Cueva u. Shannon N. Byrne (Hgg.), A Companion to the Ancient Novel, Malden, MA/Oxford, 201-216.

Genette ${ }^{3} 2010$ : Gérard Genette, Die Erzählung (übs. von Andreas Knop, mit einem Nachwort von Jochen Vogt, überprüft und berichtigt von Isabel Kranz), Paderborn.

Gill 22008: Christopher Gill, „Tragic Fragments, Ancient Philosophers and the Fragmented Self“, in: Fiona McHardy, James Robson u. David Harvey (Hgg.), Lost Dramas of Classical Athens: Greek Tragic Fragments, Exeter, 151-172.

Goldhill 2007: Simon Goldhill, „What is ekphrasis for“, Classical Philology 102, 1-19. 
Grenfell u. Hunt 1901: Bernard P. Grenfell u. Arthur S. Hunt, The Amherst Papyri: Being an Account of the Greek Papyri in the Collection of Lord Amherst of Hackney. Part II: Classical Fragments and Documents of the Ptolemaic, Roman and Byzantine Periods. With an Appendix Containing Additional Theological Fragments, London.

Grethlein 2016: Jonas Grethlein, „Minding the Middle in Heliodorus’ Ethiopica: False Closure, Triangular Foils and Self-Reflection“, Classical Quarterly 66, 316-335.

Gronewald 1979: Michael Gronewald, „Ein Fragment aus den Aithiopica des Heliodor (P. Amh $160=$ Pack² 2797)“, Zeitschrift für Papyrologie und Epigraphik 34, 19-21.

Hall 2010: Edith Hall, „Iphigenia in Oxyrhynchus and India: Greek Tragedy for Everyone“, in: Stavros Tsitsirides (Hg.), Parachoregema: Studies on Ancient Theatre in Honour of Professor Gregory M. Sifakis, Heraklion, 225-250.

Hall 2013: Edith Hall, Adventures with Iphigenia in Tauris. A Cultural History of Euripides' Black Sea Tragedy, Oxford.

Harrison u. Liapis 2013: George W.M. Harrison u. Vayos Liapis (Hgg.), Performance in Greek and Roman Theatre (Mnemosyne Suppl. 353), Leiden/Boston.

Henrichs 2011: Albrecht Henrichs, „Missing Pages: Papyrology, Genre, and the Greek Novel“, in: Dirk Obbink und Richard Rutherford (Hgg.), Culture in Pieces. Essays on Ancient Texts in Honour of Peter Parsons, Oxford, 302-322.

Hilton 1998: John Hilton, „An Ethiopian Paradox: Heliodorus, Aithiopika 4.8“, in: Richard Hunter (Hg.), Studies in Heliodorus, Cambridge, 79-92.

Hilton 1998: John Hilton, „The Sphragis of Heliodoros, Genealogy in the Aithiopika, and Julian's Hymn to King Helios“, Ágora. Estudos Clássicos em Debate 14, 195-219.

Hilton 2016: John Hilton, „Emeralds and Embassies in the Ethiopian Story of Heliodorus“, Akroterion 61, 25- 42.

Hirschberger 2001: Martina Hirschberger, „Epos und Tragödie in Charitons Kallirhoe. Ein Beitrag zur Intertextualität des griechischen Roman“, Würzburger Jahrbücher für die Altertumswissenschaft 25, 157-186.

Holzberg 1996: Niklas Holzberg, „The Genre: Novels Proper and the Fringe“, in: Gareth Schmeling (Hg.), The Novel in the Ancient World (Mnemosyne Suppl. 159), Leiden/New York/Köln, 11-28.

Hunter 2008: Richard Hunter, „Ancient Readers“, in: Tim Whitmarsh (Hg.), The Cambridge Companion to the Greek and Roman Novel, Cambridge, 261-271.

Jones 1993: Christopher J. Jones, „Greek Drama in the Roman Empire“, in: Ruth Scodel (Hg.), Theater and Society in the Classical World, Ann Arbor, 39-52.

Keyes 1922: Clinton W. Keyes, „The Structure of Heliodorus’ Aethiopica“, Studies in Philology 19, 42-51.

Klein 2004: Richard Klein, „Spectaculorum voluptates adimere... Zum Kampf der Kirchenväter gegen Circus und Theater", in: Joachim Fugmann, Markus Jankus u. a. (Hgg.), Theater, Theaterpraxis, Theaterkritik im kaiserzeitlichen Rom (Kolloquium anlässlich des 70. Geburtstages von Prof. Dr. Peter Lebrecht Schmidt, 24./25. Juli 2003, Universität Konstanz), München/Leipzig, 155-174.

Konstan 2009: David Konstan, „The Active Reader and the Ancient Novel“, in: Michael Paschalis, Stelios Panayotakis u. Gareth Schmeling (Hgg.), Readers and Writers in the Ancient Novel, Groningen, 1-17.

Kruchió 2017: Benedek Kruchió, „What Charicles Knew: Fragmentary Narration and Ambiguity in Heliodorus' Aethiopica“, Ancient Narrative 14, 175-194.

Lalanne 2008: Sophie Lalanne, „L’odyssée des héroïnes du roman grec (ler-Ille siècles après J.-C.)“, Clio 28, 121-132.

Lefteratou 2013: Anna Lefteratou, „The Myth of Iphigenia in Heliodorus' Aethiopica“, in: Marília F. Pinheiro, Anton Bierl u. Roger Beck (Hgg.), Intende, Lector. Echoes of Myth, Religion, and Ritual in the Ancient Novel, Berlin/Boston, 200-222. 
Lefteratou 2017: Anna Lefteratou, Mythological Narratives. The Bold and Faithful Heroines of the Greek Novel (MythosEikonPoiesis 8), Berlin/Boston.

Létoublon 1993: Françoise Létoublon, Les lieux communs du roman. Steréotypes grecs d'aventure et d'amour (Mnemosyne Suppl. 123), Leiden/New York/Köln.

Marino 1990: Elena Marino, „Il teatro nel romanzo: Eliodoro e il codice spettacolare“, Materiali $e$ Discussioni 25, 203-218.

Marrou 1948: Henri-Irénée Marrou, Histoire del'éducation dans l'Antiquité (Le monde romain, Bd. 2), Paris.

Mastronarde 2017: Donald J. Mastronarde, „Text and Transmission“, in: Laura McClure (Hg.), $A$ Companion to Euripides, Malden, MA/Oxford, 11-26.

Matthiessen 2002: Kjeld Matthiessen, Die Tragödien des Euripides (Zetemata 114), München. Matthiessen 2004: Kjeld Matthiessen, Euripides und sein Jahrhundert (Zetemata 119), München. Mecella 2014: Laura Mecella, „L'enigmatica figura di Eliodoro e la datazione delle Etiopiche“, Mediterraneo Antico 17, 633-658.

Menze 2017: Martin Antonius Menze, Heliodors ,klassische‘ Ekphrase. Die literarische Visualität der ,Aithiopika“ im Vergleich mit ihren Vorläufern bei Homer und Herodot sowie ihrer Rezeption bei Miguel de Cervantes (Orbis Antiquus 51), Münster.

Mignogna 1997: Elisa Mignogna, „Leucippe in Tauride (Ach. Tat. 3, 15-22): mimo e ,pantomimo“ tra tragedia e romanzo“, Materiali e discussioni per l'analisi dei testi classici 38, 225-236.

Montiglio 2013: Silvia Montiglio, Love and Providence. Recognition in the Ancient Novel, Oxford.

Morgan 1982: John R. Morgan, „History, romance and realism in the Aithiopika of Heliodoros“, Classical Antiquity 1, 221-265.

Morgan 1989: John R. Morgan, „The Story of Knemon in Heliodoros' Aithiopika“, JHS 109, 99-113.

Morgan 1991: John R. Morgan, „Reader and audiences in the ,Aithiopika“ of Heliodoros“, in: Heinz Hofmann (Hg.), Groningen Colloquia on the Novel, Bd. 4, Groningen,, 85-103.

Morgan 1994: John R. Morgan, „The Aithiopika of Heliodoros. Narrative as Riddle“, in: John R. Morgan u. Richard Stoneman, Greek Fiction. The Greek Novel in Context, London/New York, 97-113.

Morgan 1996: John R. Morgan, „Heliodoros“, in: Gareth Schmeling (Hg), The Novel in the Ancient World. (Mnemosyne. Biliotheca Classica Batava), Leiden u. a., 417-456.

Morgan 1998: John R. Morgan, „Narrative Doublets in Heliodorus' Aethiopika“, in: Richard Hunter (Hg.), Studies in Heliodorus, Cambridge, 60-78.

Morgan 2003: John R. Morgan, „Nymphs, Neighbours and Narrators: A Narratological Approach to Longus“, in: Stelios Panayotakis, Maaike Zimmerman u. Wytse Hette Keulen (Hgg.), Ancient Novel and Beyond, Leiden, 171-189.

Morgan 2012: John R. Morgan, „Heliodorus“, in: Irene de Jong (Hg.), Space in Ancient Greek Literature, Leiden/Boston, 557-578.

Mundt 2015: Felix Mundt, „Der Mensch, das Licht und die Stadt. Rhetorische Theorie und Praxis antiker und humanistischer Städtebeschreibung“, in: Therese Fuhrer, Felix Mundt u. Jan Stenger (Hgg.), Cityshaping. Constructing and Modelling Images of the City, Berlin/Boston, $176-206$.

Mundt 2016: Felix Mundt, „Jüngling trifft Mädchen - Leser trifft Welt. Herkunftsräume im griechischen Liebesroman“, in: Maximilian Benz u. Katrin Dennerlein (Hgg.), Literarische Räume der Herkunft. Fallstudien zu einer historischen Narratologie (Narratologia 51), 41-66.

Olsen 2012: Sarah Olsen, „Maculate Conception: Sexual Ideology and Creative Authority in Heliodorus' Aethiopica“, American Journal of Philology 133, 301-322.

Paulsen 1992: Thomas Paulsen, Inszenierung des Schicksals: Tragödie und Komödie im Roman des Heliodor, Trier.

Pletcher 1998: James Pletcher „Euripides in Heliodoros’ Aithiopika 7-8“, in: Heinz Hofmann u. Maaike Zimmerman (Hgg.), Groningen Colloquia on the Novel, Bd. 9, Groningen, 17-27. 
Potter 2004: David S. Potter, The Roman Empire at Bay: AD 180-395, London/New York.

Puk 2014: Alexander Puk, Das römische Spielwesen in der Spätantike (Millennium Studies 48), Berlin/Boston.

Reeve 1988: Michael D. Reeve, „Conceptions“, Proceedings of the Cambridge Philological Society 35, 81-112.

Rocca 1976: Rosanna Rocca, „Eliodoro e i due Ippoliti euripidei“, Materiali e contributi per la storia della narrativa greco-latina $1,23-31$.

Ross 2014: Alan J. Ross, „Syene as Face of Battle: Heliodorus and Late Antique Historiography“, Ancient Narrative 12, 1-26.

Schauer 2002: Markus Schauer, Tragisches Klagen. Form und Funktion der Klagedarstellung bei Aischylos, Sophokles und Euripides (Classica Monacensia. Münchener Studien zur klassischen Philologie 26), Tübingen.

Scourfield 2007: John H.D. Scourfield, „Textual Inheritances and Textual Relations in Late Antiquity“, in: John H.D. Scourfield (Hg.), Texts and Culture in Late Antiquity. Inheritance, Authority, and Change, Swansea, 1-32.

Scourfield 2010: John H.D. Scourfield, „Chaereas, Hippolytus, Theseus: Tragic Echoes, Tragic Potential in Chariton", Phoenix 64, 291-313.

Seidensticker 1982: Bernd Seidensticker, Palintonos Harmonia. Studien zu komischen Elementen in der griechischen Tragödie (Hypomnemata 72), Göttingen.

Smith 2007: Steven D. Smith, „Wonders beyond Athens: Reading the ,Phaedra“ Stories in Apuleius and Heliodoros“, in: Michael Paschalis, Stavros Frangoulidis, Stephen Harrison u. Maaike Zimmerman (Hgg.), The Greek and the Roman Novel. Parallel Readings (Ancient Narrative Supplementum 8), Groningen, 219-237.

Stephens 2008: Susan Stephens, „Cultural Identity“, in: Tim Whitmarsh (Hg.), The Cambridge Companion to the Greek and Roman Novel, Cambridge, 56-71.

Tagliabue 2015: Aldo Tagliabue, „Heliodorus's Aethiopica and the Odyssean Mnesterophonia: An Intermedial Reading“, Transactions of the American Philological Association 145, 445-468.

Telò 2011: Mario Telò, „The Eagle's Gaze in the Opening of Heliodorus' Aethiopica“, American Journal of Philology 132, 581-632.

Tilg 2010: Stefan Tilg, „Mythos, Fiktion, Geschichte: Ein Beitrag zum ,Realismus“ der antiken Romane“, Ancient Narrative 9, 37-52.

Walden 1984: John W.H.Walden, „Stage-Terms in Heliodorus's Aethiopica“, Harvard Studies in Classical Philology 5, 1- 43.

Webb 2007: Ruth Webb, „Rhetoric and the Novel: Sex, Lies and Sophistic“, in: Ian Worthington (Hg.), A Companion to Greek Rhetoric, Malden, MA/Oxford, 526-541.

Webb 2009: Ruth Webb, Ekphrasis, Imagination and Persuasion in Ancient Rhetorical Theory and Practice, Farnham u. a.

Whitmarsh 1998: Tim Whitmarsh, „The Birth of a Prodigy: Heliodorus and the Genealogy of Hellenism“, in: Richard Hunter (Hg.), Studies in Heliodorus (Cambridge Philological Society. Supplementary Volume 21), Cambridge, 93-124.

Whitmarsh 1999: Tim Whitmarsh, „The Writes of Passage. Cultural Initiation in Heliodorus' Aethiopica“, in: Richard Miles (Hg.), Constructing Identities in Late Antiquity, London, 16-40.

Whitmarsh 2011: Tim Whitmarsh, Narrative and Identity in the Ancient Greek Novel. Returning Romance, Cambridge.

Whitmarsh 2018: Tim Whitmarsh, Dirty Love: The Genealogy of the Ancient Greek Novel, Oxford.

Winkler 1982: John J. Winkler, „The Mendacity of Kalasiris and the Narrative Strategy of Heliodoros' Aithiopika“, Yale Classical Studies 27, 93-158.

Winkler 2002: Martin M. Winkler, „The Cinematic Nature of the Opening Scene of Heliodorus' Aithiopika“, Ancient Narrative 1,161-184. 
Zeitlin 2013: Froma I. Zeitlin, „Landscapes and Portraits. Signs of the Uncanny and Illusions of the Real", in Michalis Paschalis u. Stelios Panayotakis (Hgg.), The Construction of the Real and the Ideal in the Ancient Novel (Ancient Narrative Supplementum 17), 61-87, Groningen. 